Plastic Surgery : A short course of lecture-demonstrations is being arranged, to be given at the Hammersmith Hospital, by Sir Harold Gillies, Mr. MacIndoe and Mr. Kilner. Details will be circulated shortly.

Technique of Operations: A series of demonstrations is being arranged. Details will be circulated shortly.

Demonstrations in (Advanced) Medicine and Surgery; A series of weekly demonstrations is being arranged. Details will be circulated shortly.

A Guide Book, giving details of how to reach the various London Hospitals by tube, tram, or bus, can be obtained from the Fellowship. Price 6d. (Members and Associates, 3d.).

\title{
SINUSITIS AS A CAUSE OF TONSILLITIS.
}

\author{
BY BEDFORD RUSSELL, F.R.C.s., \\ Surgeon-in-Charge, Throat Department, St. Bart's Hospital.
}

Although the existence of sinus-infection has long since been recognized, medical men whose work lies chiefly in the treatment of disease in the nose, throat and ear are frequently struck with the number of cases of sinusitis which have escaped recognition even in the presence of symptoms and signs which should have given rise at least suspicion of such disease. The explanation of the failure to recognize any but the most manifest cases of sinusitis lies, 1 think, in the extreme youth of this branch of medicine; for although operations upon the nose were undoubtedly performed thousands of years ago, it was not until the adoption of cocaine about forty years ago that it was possible even to examine the nasal cavities really critically. The first twenty years of the cocaine epoch were marked by great activity in nose work, and rapid progress was made in this country, notably by St. Clair Thomson, Tilley, Dundas-Grant and Watson Williams; also, of course, in America and on the Continent. The intensive study of ear-troubles was already under way, but was treated rather as a separate affair till it was gradually borne in upon the profession that the ear was actually a nasal sinus, not only from embryological considerations, but in many aspects of its behaviour in disease. Indeed, the best way to regard the ear is as a nasal sinus across the middle of which Nature has for no obvious reason chosen to thrust the organ of Hearing. The lining of the ear (furnished even to the cells of the mastoid process with the cilia always found elsewhere in the nose), persists in carrying on with its usual nasal functions, in spite of the mechanical difficulties caused by the intrusion of the sense-organ. Hearing often suffers, owing to the inflammations resulting from infections in the middle ear; and this is the price it has to pay for sharing a corner of the head with a structure which is still a nasal sinus, and which therefore cannot escape taking part in many of the responsibilities and risks undertaken by the rest of the sinuses in their adventures with inspired micro-organisms.

The functions of the sinuses are doubtless quite numerous, and some of them are quite obvious to us. They serve to lighten the front of the head, thus helping to balance 
it upon the neck; and, by expanding the bone in the mastoid processes and the maxilla, they provide light, strong projections for muscular attachments. They are also responsible, by vibrating during speech, for the overtones which differentiate their owner's voice from all others; and they further help in building up his personality by determining the shape of much of his face. But it is also possible that they have a most important function in connection with immunity. It has been shown that the mouths of some of the sinuses are so shaped, that when air is drawn past them an eddy is created within the sinus-cavity, connoting a changing of a portion of their contained air, together with a slowing-down of the speed of the air which eddies into them. And it seems possible that there may be deposited within the sinuses a sample of whatever micro-organisms each breath contains.

This is merely a guess. But we have more definite knowledge of the ultimate fate of some inspired substances, in whatever part of the nose they happen to be arrested by the mucus moistening the lining membrane. Yates showed that Indian ink introduced into the antra is swept by the cilia out through the ostium, back into the post-nasal space, and finally to the ossophagus. On its way, the thin black line of Indian ink particles is seen to send a sort of ana-branch which runs forward as far as the tonsil and then rejoins the parent stream. So that the tonsil has a sort of perquisite-the privilege of sampling everything that comes down from the nose; and it seems that we have in this fact both a hint as to one of the functions of the tonsil, and an explanation of why we so often see a unilateral tonsillar inflammation associated with an antral or ethmoidal infection upon the same side. During the loose stage of a cold, one can often catch sight of a thin streak of muco-pus-the homologue of the black streak of Indian inkcoming forward over the posterior pillar of the fauces on to the tonsil : and one feels that the tonsil is sometimes blamed and removed for an inflammatory protest at the quality of the nasal sample it receives, when the unfortunate organ should rather be rewarded for the valour of its part in a remedial effort. (I, personally, have small doubt that the tonsil is concerned in the manufacture, and ultimate introduction via its lymphatics into the blood-stream, of antibodies appropriate to the infections brought to it by the above-described mechanisms.)

Viewed in this way, we may legitimately regard a tonsillitis very often as merely constituting a symptom of sinusitis; and a searching examination of the sinuses, especially of the posterior ethmoids, in all cases of tonsillitis, shows the existence of sinusitis in a surprisingly large proportion of cases.

It is very natural that with the development of methods of examining the throat and nose, the condition of the tonsil rather than the sinuses should have been the first to receive attention at the hands of the profession. For one thing, it is easy to see without the need for developing special skill at manipulating a mirror; and, owing to the fact that it is a mobile organ, it is painful when it is inflamed; and this leads to a pleasing unanimity between doctor and patient in a belief as to the whereabouts of the infective stronghold. Moreover, as tonsillectomy is usually followed by some improvement in health, and occasionally by a really dramatic cure of symptoms, it is small wonder that what is really the villain of the piece often escapes attention till later on. If the tonsil were the real primary focus of naso-pharyngeal disease, those of us who see large numbers of out-patients with catarrhal complaints would expect that the majority of them would possess tonsils; and this was so before the War. But the wave of 
tonsillectomania which followed the adoption of Sluder's guillotine-operation in about I9Io has seen to it that very few people suffering from sore throats, colds, glands in the neck, and so forth, have escaped tonsil operations.

During the earlier years it was not possible to assess the value of tonsillectomy because operations varied between tonsillectomy at one end of the scale and removal .of the tonsil-bearing area at the other; and any persistence of symptoms after operation was ascribed to imperfections in technique, often quite rightly. But with the development of skill in anæsthesia, satisfactory tonsillectomy, whether by dissection or guillotine, became the rule rather than the exception; and it has become possible during the last decade to assess the therapeutic value of the operation.

The disorders which it was hoped to relieve by removal of the tonsils covered a very wide range ; and laryngologists are still constantly having cases referred to them for the relief of colds, sore throats, mouth-breathing, rheumatism, chorea, baldness, acidosis, nephritis, iritis, and indeed all those conditions which may be reasonably attributed to absorption from any septic focus. (A group-name, by the way, is badly needed for these conditions. "Endogenous" is not quite descriptive enough ; and the hybrid "focogenic" would repel classical scholars.)

And it is not to be denied that very numerous cases of any of these types are constantly being relieved or even dramatically cured by tonsillectomy, and especially by the removal of tonsillar remains. But it is also true that very numerous patients present themselves every day in our throat departments from whom unhealthy tonsils have been elegantly removed, and who nevertheless still complain of the very disorders for whict this operation was performed. One of the commonest disappointments is the persistence. of enlargement of glands in the neck after tonsillectomy. These glands help to drai the nasal sinuses, as well as the tonsils; and their persistence after tonsillectomy reminds us of this neglected fact. Evidently, therefore, in these disappointing cases the essential focus is still present ; and it becomes desirable to compare these patients with those who have duly benefited from tonsillectomy. Assuming that the focus was indeed nasopharyngeal and that there be no infected teeth, it will be found that they all have signs or at least a definite history pointing to a sinusitis. And if our reading of the physiology of these regions be correct, it would surely have been more logical to treat the nose-trouble before paying attention to the tonsillar condition. Moreover, it ought to be expected that if a sinusitis can be relieved, the tonsils should respond by sinking back into their former obscurity, shouldering afresh their former humdrum responsibility-the assessment of the daily and hourly sample of inspired organisms brought down by the ciliary stream from the nasal passages. This is indeed the case in practice; and it is surprising how far gone in iniquity a tonsil can be and yet recover its normal habit after the drainage of, say, an infected antrum. I would go so far as to say that one can expect immediately to put an end to a series of tonsillar attacks by adequate ventilation and drainage of all the offending sinuses. And it is essential that the word " all" be noted ; for it is almost a truism in nose-work that a nasal operation, in which an infected cell of importance is missed, is likely to be followed by a flare-up-a flare-up which does not confine itself too the nose, but which is often followed by an acute tonsillitis as well. Tonsillitis following a simple septum-resection is due to the operation of just this principle, and often provokes the tardy disclosure of a latent sinusitis. And this brings me to the most important portion of my remarks, dealing with the frequency of sinusitis the iniquity thereof, and the question of diagnosis. 
When does a "Cold" become a "Sinusitis"?-With an ordinary cold there is an increased secretion of mucus, or of muco-pus, not only from the nasal cavities proper, but probably from every nasal sinus, not excluding the mastoid antrum; and successful resolution connotes a removal of the secretion by ciliary activity, as fast as it is produced if the cold be a mild one, the sinuses never quite filling up; or somewhat slower than it is produced if the cold be a heavy one, the sinuses in this case filling up with phlegm for a day or so. But if the ciliary removal of secretions is prevented by some structural irregularity such as a septal spur blocking the mouth of a sinus, or (far more serious) if the cilia are temporarily paralysed by the toxicity of the virus, as in an influenza cold, then the condition begins to merit the title of Sinusitis and leads to an important invasion of the lining membrane with organisms.

Transient sinusitis is excessively frequent, and will as a rule yield to such simple methods as inhalations of menthol or spraying with a one per cent. solution of Ephedrine. But a lasting infection of the sinuses also is much more frequent than is usually recognized, especially during an influenza epidemic ; and one is beginning to date nearly all cases of (non-dental) chronic sinusitis back to one of the well-known influenza epidemics, notably those in I918, I929 and r932. It is not yet possible, of course, to say how much chronic sinusitis is going to result from this current epidemic, but my impression so far is that while there has been a tendency to sinusitis in almost all the victims, yet the sinuses have cleared up quite rapidly except in cases where the mucosal lining has been damaged in one of the above-mentioned "vintage" years. Other diseases which may initiate the long train of local and general disorders resulting from sinusitis are of course the exanthemata and similar diseases. I had occasion recently to examine a child of ro who had had an acute otitis media upon the left side at the beginning of this winter. By translumination it was found that the antra were very dark indeed though the frontals were clear. The otitis media, which had followed upon a cold of a few days duration, necessitated puncture of the left membrana tympani. The left tonsil was observed to be much larger and more inflamed than its fellow, and the mucosa lining the left nasal passage was bright red and plastered with muco-pus, that upon the other side being but little redder than normal. The trouble cleared up very rapidly with an Ephedrine spray and an alkaline nose-lotion, and he remained well until an attack of vomiting and left otalgia, with pyrexia, this January. A radiogram now showed that there was a greatly thickened lining to the left antrum, the left posterior ethmoids and sphenoids. The nose was apparently almost free from pus, but by posterior rhinoscopy, a thin film of pus was seen extending from the posterior nares on to the adenoids, and they and the tonsils were much swollen. In view of the persistence of the sinusitis, the history was gone into, and it transpired that the child had been healthy until whooping-cough in January, I93I, shortly after which the family doctor had condemned the adenoids and tonsils owing to nasal stuffiness.

This case comprises several points of interest, the first of which is the importance, in naso-pharyngeal troubles, of the past history. When examining a case, one should always ask not only "How long?" but "What began the trouble?"

The next is that (as I personally believe) middle ear inflammations are to be regarded as a part of a sinusitis, and not usually as a result of inflammation in tonsils and adenoids. These organs rather are inflamed in consequence of the sinusitis which is involving the middle ear. If, of course, the adenoids swell very much, then 
there is no doubt whatever that they block the eustachian tubes and set up otitis media. But one sees numerous cases of otitis media after removal of every trace of adenoids $\underset{\mathbb{\Phi}}{\overline{0}}$ and tonsils; so that they cannot be the essential causal.agent.

Another point is the value of translumination. Before the War one used to hear this undeniably useful test condemned as practically worthless; and the reason for the diversity of opinion is, I think, that the test used first to be employed during the period when "sinusitis" meant an actual empyema of a sinus : if antral puncture produced no $\bar{c}$ pus, there was held to be no sinusitis. And if after translumination had shown a dull $\mathbb{\Omega}$ antrum, proof-puncture produced no discharge, there was another nail in the coffin os of translumination as an indicator of sinusitis.

The commonest cause of dimness of an antrum or frontal sinus is hyperamia; and it may be of great importance to be able to record the presence of this universal sign of active inflammation. Supposing for instance that a patient who has translucent 8 antra when well, shows a dimness of the left antrum whenever he has a cold; one can $\frac{3}{6}$ hardly disregard so definite a sign. It usually means that somewhere in that side of the nose (not usually in the antrum itself, strangely enough, but rather in a neighbouring ${ }^{\circ}$ ethmoidal cell) is a collection of muco-pus which cannot escape, or a thickened-which $ᄋ$ is to say an infected-lining; and his repeated colds are most probably not caught from other people, but are merely an expression of the nose's periodical attempt to cope with $\frac{3}{9}$ the local infection. Again, differential translumination between the frontals and the $\frac{\sigma}{3}$ antra may give important information. Supposing that no light is transmitted by $\overrightarrow{0}$ frontals or antra ; one may merely be dealing with a person whose bones are thick, and whose sinuses are small ones. But if during each cold the frontals are clear, then we. may the more reasonably take notice of dull antra as an indication of the chief strongholg of the recurring infection.

One more very important example of the use of translumination before considering radiography in sinusitis. A boy of $\mathrm{I} 7$ had had a widespread chronic sinusitis, but as $\frac{2}{\vec{P}}$ the left antrum was the only dull sinus, it was drained, and soon cleared up. During $\cong$ and after an influenza epidemic some months later the boy became much debilitated and had lumbago, there being no physical signs obvious to the family doctor by which to localise the suspected focus of absorption. Translumination, however, showed that the left antrum, which had previousIy become translucent, was now quite opaque again, even after syringeing out, the opacity obviously therefore being due to the hyperæmia caused by a lighting-up of the old infection which had lain dormant in the sinus-lining. The whole trouble cleared up speedily upon diathermy to the antrum, injections of manganese, sunlight, \&c. I suggest that just as every physician should learn to interpret ophthalmoscopic findings, so he should be able to assess the much more easily? interpreted findings by translumination (a test, by the way, which he should use in o apparent health as well as in obvious disease).

The interpretation of radiograms is a specialist's job, but one or two points may స్ల్ interest all graduates. One of the chief uses of radiograms is in furnishing evidence about those much-neglected but very important sinuses which do not lend themselveso to translumination-the ethmoids and sphenoids. These probably give rise between them to more troubles than ever do the antra and frontals, but are ignored by the average medical man because of the great difficulty in examining them. Within the last two years the general practitioner has been able to avail himself of this (at last very 
reliable) method of examining sinuses.' He can so help to exclude this commonly unsuspected cause of rheumatism, eye-trouble, headache and chronic dyspepsia, and may thus obviate an unnecessary visit to a rhinologist.

Another way in which they help is in the demonstration of the presence of polypi (and therefore of latent infection) in the antral lining. For some unexplained reason, many antral polypi which are quite translucent in the dark room show up very clearly by X-rays.

In conclusion, it should be realised that while the complete cure of a chronic sinusitis may call for operative measures, yet much relief may usually be afforded by less drastic means; and that in acute sinusitis, with the exception of really fulminating cases, non-operative means should be the method of choice. The intelligent use of cocaine is the greatest stand-by at our command in acute disease of the nose, the drug preferably being "protected" by some adjuvant vaso-constrictor such as ephedrine ; and there would be fewer cases of chronic sinusitis if the profession would realise what a potent weapon we possess in cocaine for the relief of obstruction both to the airway and to the exits from the sinuses.

In chronic sinusitis, effective measures of a non-operative nature are harder to come by: but it should be recognized that the routine use of nasal lotions may be largely stultified by failure to ensure that the lotions attain to the chinks and crannies for which they are intended ; the desired end may be furthered by occasional shrinking of the congested nasal mucus by vaso-constrictors, as a prelude to the sniffing or spraying of the usual alkaline nose-wash. Another quite potent weapon is the intramuscular injection of collosol manganese, which, in a fairly well-ventilated nose, is especially likely to help in clearing up the low-grade catarrh which so often follows influenza and for which vaccines are often ineffective.

\title{
THE SIGNIFICANCE OF BLEEDING AS A SYMPTOM IN GYN ECOLOGY.
}

\author{
BY MARTIN OLDERSHAW, M.D.LOND., F.R.C.S.ENG., \\ Hon. Surgeon to In-and Out-patients, the Hospital for Women, Soho Square, W.1.
}

IN general practice, gynæcological conditions form a large and important group of cases. These patients are often reluctant to seek advice unless some urgent symptom is present, and in many cases the symptom which first sends them to a doctor is vaginal bleeding. Such bleeding may be due to a large number of causes, many of them trivial, others extremely serious, and in this lecture I have attempted to outline the various conditions which may be present, and to describe the way in which the problem of diagnosis may be tackled.

It is convenient to arrange cases into three groups according to age, namely, "Before Puberty," " During Reproductive Life," and "After the Menopause."

Before Puberty.-Vaginal bleeding is rare in children and is usually due to trauma, such as scratching, attempted rape, or the insertion of a foreign body, for example, a pencil. If bleeding occurs in the absence of any injury, thorough examination is required,

${ }^{1}$ Hodgson, British Medical Journal, January 7, 1933. 